\title{
A GENERALIZATION OF INVERSE SCHWARZ'S INEQUALITY
}

\section{GOU-SHENG YANG AND KAI-YU TENG}

\section{Introduction}

In 1915, Frank and Pick [4] proved the following inverse Schwarz's inequality: If $u$ and $v$ are nonnegative and concave functions on the interval $[0,1]$, then

$$
\int_{0}^{1} u(t) v(t) d t \geq \frac{1}{2}\left(\int_{0}^{1} u^{2}(t) d t\right)^{\frac{1}{2}}\left(\int_{0}^{1} v^{2}(t) d t\right)^{\frac{1}{2}} .
$$

Bellman [2] generalized (1.1) into the following form:

$$
\int_{0}^{1} u(t) v(t) d t \geq \frac{(A+1)^{\frac{1}{A}}(B+1)^{\frac{1}{B}}}{6}\left(\int_{0}^{1} u^{A}(t) d t\right)^{\frac{1}{A}}\left(\int_{0}^{1} v^{B}(t) d t\right)^{\frac{1}{B}},
$$

where $1<A, B<\infty$ with $\frac{1}{A}+\frac{1}{B}=1$.

We note that the inequality (1.1) is the special case of (1.2) when $A=B=2$.

Recently, Alzer [1] established the following generalization of (1.1):

$$
\int_{0}^{1} u^{p}(t) v^{q}(t) d t \geq\left(\frac{2 \sqrt{(2 p+1)(2 q+1)}}{(p+1)(q+1)}-1\right)\left(\int_{0}^{1} u^{2 p}(t) d t\right)^{\frac{1}{2}}\left(\int_{0}^{1} v^{2 q}(t) d t\right)^{\frac{1}{2}}
$$

where $p>0, q>0$.

We note that the inequality (1.1) is the special case of (1.3) when $p=q=1$.

Our aim of this article is to generalize the inequality (1.3) in several forms.

Received June 25, 1991. 


\section{Preliminaries}

Lemma 1. (Alzer [1] ) Let $u, v$ and $w$ be integrable functions on $[0,1]$ and $\int_{0}^{1} w^{2}(t) d t \neq 0$. Then

$\frac{1}{2}\left[\left(\int_{0}^{1} u^{2}(t) d t\right)^{\frac{1}{2}}\left(\int_{0}^{1} v^{2}(t) d t\right)^{\frac{1}{2}}+\int_{0}^{1} u(t) v(t) d t\right] \geq \frac{\int_{0}^{1} u(t) w(t) d t \cdot \int_{0}^{1} v(t) w(t) d t}{\int_{0}^{1} w^{2}(t) d t}$.

Lemma 2. ( Berwald [3] ) Let $u$ be nonnegative and concave on $[0,1]$. If $0<p \leq q$, then

$$
\left[(q+1) \int_{0}^{1} u^{q}(t) d t\right]^{\frac{1}{q}} \leq\left[(p+1) \int_{0}^{1} u^{p}(t) d t\right]^{\frac{1}{p}} .
$$

Lemma 3. If $\alpha>-1,0<p \leq q$ and $u$ is nonnegative such that $u\left(t^{\frac{1}{1+\alpha}}\right)$ is concave on $[0,1]$, then

$$
\left[(\alpha+1)(q+1) \int_{0}^{1} u^{q}(t) t^{\alpha} d t\right]^{\frac{1}{q}} \leq\left[(\alpha+1)(p+1) \int_{0}^{1} u^{p}(t) t^{\alpha} d t\right]^{\frac{1}{p}} .
$$

Proof. Using Lemma 2, we have

$$
\left[(q+1) \int_{0}^{1} u^{q}\left(t^{\frac{1}{1+\alpha}}\right) d t\right]^{\frac{1}{q}} \leq\left[(p+1) \int_{0}^{1} u^{p}\left(t^{\frac{1}{1+\alpha}}\right) d t\right]^{\frac{1}{p}}
$$

for $0<p \leq q$.

With the change of variable $x=t^{\frac{1}{1+\alpha}}$, we have the desired inequality (2.1).

Remark. Lemma 2 is the special case of Lemma 3 when $\alpha=0$.

Theorem 1. Let $\alpha>-1 ; p, q>0$. If $u$ and $v$ are nonnegative such that $u\left(t^{\frac{1}{1+\alpha}}\right)$ and $v\left(t^{\frac{1}{1+\alpha}}\right)$ are concave on $[0,1]$, then we have

$$
\begin{aligned}
\int_{0}^{1} u^{p}(t) v^{q}(t) d t \geq & {\left[\frac{2}{(p+1)(q+1)}-\frac{1}{\sqrt{(2 p+1)(2 q+1)}}\right](A+1)^{\frac{p}{A}}(B+1)^{\frac{q}{B}} } \\
& \cdot(\alpha+1)^{\frac{p}{A}+\frac{q}{B}-1}\left(\int_{0}^{1}[u(t)]^{A} t^{\alpha} d t\right)^{\frac{p}{A}}\left(\int_{0}^{1}[v(t)]^{B} t^{\alpha} d t\right)^{\frac{q}{B}},
\end{aligned}
$$


for $p \leq A \leq 2 p, q \leq B \leq 2 q ;$ and

$$
\begin{aligned}
\int_{0}^{1} u^{p}(t) v^{q}(t) t^{\alpha} d t \geq & {\left[\frac{2(A+1)^{\frac{p}{A}}(B+1)^{\frac{q}{B}}}{(p+1)(q+1)}-1\right](\alpha+1)^{\frac{p}{A}+\frac{g}{B}-1} } \\
& \cdot\left(\int_{0}^{1}[u(t)]^{A} t^{\alpha} d t\right)^{\frac{p}{A}}\left(\int_{0}^{1}[v(t)]^{B} t^{\alpha} d t\right)^{\frac{q}{B}}
\end{aligned}
$$

for $A \geq 2 p, B \geq 2 q$.

Proof. If we replace $u$ and $v$ by $u^{p}(t) t^{\frac{\alpha}{2}}$ and $v^{q}(t) t^{\frac{\alpha}{2}}$, respectively, in Lemma 1 , and set $w(t)=t^{\frac{\alpha}{2}}$, then we have the inequality

$$
\begin{aligned}
& \frac{1}{2}\left[\left(\int_{0}^{1} u^{2 p}(t) t^{\alpha} d t\right)^{\frac{1}{2}}\left(\int_{0}^{1} v^{2 q}(t) t^{\alpha} d t\right)^{\frac{1}{2}}+\int_{0}^{1} u^{p}(t) v^{q}(t) t^{\alpha} d t\right] \\
\geq & (\alpha+1)\left(\int_{0}^{1} u^{p}(t) t^{\alpha} d t\right)\left(\int_{0}^{1} v^{q}(t) t^{\alpha} d t\right) .
\end{aligned}
$$

Since $0<p \leq A, 0<q \leq B$, using Lemma 3 to the right hand side of (3.3), we have

$$
\begin{aligned}
\int_{0}^{1} u^{p}(t) v^{q}(t) t^{\alpha} d t \geq & {\left[\frac{2(A+1)^{\frac{p}{A}}(B+1)^{\frac{q}{B}}}{(p+1)(q+1)}(\alpha+1)^{\frac{p}{A}+\frac{q}{B}-1} \cdot\left(\int_{0}^{1} u^{A}(t) t^{\alpha} d t\right)^{\frac{p}{A}}\right.} \\
& \left.\cdot\left(\int_{0}^{1} v^{B}(t) t^{\alpha} d t\right)^{\frac{q}{B}}\right]-\left(\int_{0}^{1} u^{2 p}(t) t^{\alpha} d t\right)^{\frac{1}{2}}\left(\int_{0}^{1} v^{2 q}(t) t^{\alpha} d t\right)^{\frac{1}{2}} .
\end{aligned}
$$

Now if $A \leq 2 p, B \leq 2 q$ using Lemma 3 again to the last term of (3.4), we have

$$
\begin{aligned}
\left(\int_{0}^{1} u^{2 p}(t) t^{\alpha} d t\right)^{\frac{1}{2}}\left(\int_{0}^{1} v^{2 q}(t) t^{\alpha} d t\right)^{\frac{1}{2}} \leq & \left(\frac{(A+1)^{\frac{p}{A}}(B+1)^{\frac{q}{B}}(\alpha+1)^{\frac{p}{A}+\frac{q}{B}-1}}{\sqrt{(2 p+1)(2 q+1)}}\right) \\
& \cdot\left(\int_{0}^{1} u^{A}(t) t^{\alpha} d t\right)^{\frac{p}{A}}\left(\int_{0}^{1} v^{B}(t) t^{\alpha} d t\right)^{\frac{q}{B}} .
\end{aligned}
$$

The inequality (3.1) follows immediately from (3.4) and (3.5).

If $A \geq 2 p, B \geq 2 q$, an application of Hölder inequality yields

$$
\begin{aligned}
& \left(\int_{0}^{1} u^{2 p}(t) t^{\alpha} d t\right)^{\frac{1}{2}}\left(\int_{0}^{1} v^{2 q}(t) t^{\alpha} d t\right)^{\frac{1}{2}} \\
\leq & (\alpha+1)^{\frac{p}{A}+\frac{q}{B}-1} \cdot\left(\int_{0}^{1} u^{A}(t) t^{\alpha} d t\right)^{\frac{p}{A}}\left(\int_{0}^{1} v^{B}(t) t^{\alpha} d t\right)^{\frac{a}{B}} .
\end{aligned}
$$


The inequality (3.2) follows from (3.4) and (3.6).

Corollary 1. Let $\alpha \geq 0, p, q>0$. If $u$ and $v$ are nonnegative, concave and increasing on $[0,1]$, then (3.1) and (3.2) hold.

Proof. Let $g(t)=t^{\frac{1}{1+\alpha}}, t \in[0,1]$. Then $g$ is concave on $[0,1]$. If $t_{1}, t_{2} \in$ $[0,1]$, and let $f(t)=u\left(t^{\frac{1}{1+\alpha}}\right), t \in[0,1]$. Then

$$
\begin{aligned}
f\left(a t_{1}+b t_{2}\right)=u\left(g\left(a t_{1}+b t_{2}\right)\right) & \geq u\left(a g\left(t_{1}\right)+b g\left(t_{2}\right)\right) \\
& \geq a u\left(g\left(t_{1}\right)\right)+b u\left(g\left(t_{2}\right)\right) \\
& =a f\left(t_{1}\right)+b f\left(t_{2}\right) .
\end{aligned}
$$

for all $a, b \in[0,1]$ with $a+b=1$.

This shows that $u\left(t^{\frac{1}{1+\alpha}}\right)$ is concave on $[0,1]$. Similarly, $v\left(t^{\frac{1}{1+\alpha}}\right)$ is concave on $[0,1]$. The results then follows from Theorem 1 .

Corollary 2. Let $u(t)$ and $v(t)$ be nonnegative and concave on $[0,1]$ and $0<p \leq A, 0<q \leq B$. Then we have

$$
\begin{aligned}
\int_{0}^{1} u^{p}(t) v^{q}(t) d t \geq & {\left[\frac{2}{(p+1)(q+1)}-\frac{1}{\sqrt{(2 p+1)(2 q+1)}}\right](A+1)^{\frac{p}{A}}(B+1)^{\frac{q}{B}} } \\
& \cdot\left(\int_{0}^{1}[u(t)]^{A} d t\right)^{\frac{p}{A}}\left(\int_{0}^{1}[v(t)]^{B} d t\right)^{\frac{q}{B}}
\end{aligned}
$$

for $A \leq 2 p, B \leq 2 q ;$ and

$$
\int_{0}^{1} u^{p}(t) v^{q}(t) d t \geq\left[\frac{2(A+1)^{\frac{p}{A}}(B+1)^{\frac{q}{B}}}{(p+1)(q+1)}-1\right]\left(\int_{0}^{1}[u(t)]^{A} d t\right)^{\frac{p}{A}}\left(\int_{0}^{1}[v(t)]^{B} d t\right)^{\frac{q}{B}},
$$

for $A \geq 2 p, B \geq 2 q$.

Proof. This is the case $\alpha=0$ of Theorem 1 .

Remarks.

1. Inequality (1.3) is the special case of Corollary 2 by taking $A=2 p, B=2 q$.

2. If we let $p=q=1$, then Corollary 2 becomes :

$$
\int_{0}^{1} u(t) v(t) d t \geq \frac{(A+1)^{\frac{1}{A}}(B+1)^{\frac{1}{B}}}{6}\left(\int_{0}^{1} u^{A}(t) d t\right)^{\frac{1}{A}}\left(\int_{0}^{1} v^{B}(t) d t\right)^{\frac{1}{B}},
$$


for $1 \leq A \leq 2,1 \leq B \leq 2$; and

$$
\int_{0}^{1} u(t) v(t) d t \geq\left[\frac{(A+1)^{\frac{1}{A}}(B+1)^{\frac{1}{B}}}{2}-1\right]\left(\int_{0}^{1} u^{A}(t) d t\right)^{\frac{1}{A}}\left(\int_{0}^{1} v^{B}(t) d t\right)^{\frac{1}{B}},
$$

for $A \geq 2, B \geq 2$.

\section{References}

[1] H. Alzer, "On an integrall inequality of R. Bellman", Tamkang J. Math. Vol. 22, No. 2 (1991) 18\%-191.

[2] R. Bellman, "Converses of Schwarz's inequality", Duke Math. J. 23 (1956), 429-434.

[3] L. Berward, "Verallgemeinerung eines Mittelwertsatzes von J. Favard fur Positive Konkave Funktionen", Acta Math. 79 (1947), 17-37.

[4] P. Frank and G. Pick, "Distanzchätzungen im Functionenraum : I", Math. Anr. 78 (1915), 354-375.

Department of Mathematics, Tamkang University, Tamsui, Taiwan 25137. 